\title{
Expression of cyclooxygenase-2 and mucin 1 in colorectal cancer
}

\author{
MAŁGORZATA SZLENDAK ${ }^{1,2}$, ROBERT SITARZ ${ }^{1,3,4}$, MONIKA BERBECKA $^{1}$, JERZY MIELKO ${ }^{2}$, \\ FOLKERT MORSINK $^{4}$, RYSZARD MACIEJEWSKI ${ }^{1}$, G. JOHAN A. OFFERHAUS ${ }^{4}$ and WOJCIECH P. POLKOWSKI ${ }^{2}$ \\ Departments of ${ }^{1}$ Human Anatomy and ${ }^{2}$ Surgical Oncology, Medical University of Lublin; \\ ${ }^{3}$ Department of Surgery, Center of Oncology of The Lublin Region St. Jana z Dukli, Lublin 20-090, Poland; \\ ${ }^{4}$ Department of Pathology, University Medical Center, Utrecht 3508 GA, The Netherlands
}

Received January 22, 2020; Accepted July 9, 2020

DOI: $10.3892 / \mathrm{mco} .2020 .2122$

\begin{abstract}
In colorectal cancer (CRC), pathological factors that correlate with negative prognosis include, among others, overexpression of cyclooxygenase- 2 (COX-2) and abundant expression of mucin 1 (MUC1). COX-2 overexpression may therefore be associated with MUC1 overexpression. The aim of the present study was to investigate the possible correlation between COX-2 and MUC1 expression and to assess the correlation between their individual expression and the clinicopathological features of patients, paying particular attention to survival. The following data was collected from the 170 patients with CRC included in the present study: Age, sex, tumour localization, disease stage and survival. Tumour samples were immunostained with antibodies against COX-2 and MUC1. Protein expression was scored, relative to reference staining, and correlated with the clinicopathological data of patients. The results revealed no correlation between the expressions of COX-2 and MUC1, or with any of the studied clinicopathological variables. In addition, the expression of the two proteins were not associated. Neither of the proteins demonstrated prognostic value for survival. The present study did not confirm a direct relationship between the expressions of COX-2 and MUC1, or between the expression of either protein and the clinicopathological features of patients, including survival.
\end{abstract}

\section{Introduction}

According to epidemiological data gastrointestinal cancer is one of the largest problems within the field of oncology (1). In 2018, colorectal cancer (CRC) was the cause of 881,000 deaths (2). However, treatment and management of CRC have considerably changed over the last years.

Correspondence to: Dr Małgorzata Szlendak, Department of Human Anatomy, Medical University of Lublin, 4 Jaczewskiego, Lublin 20-090, Poland

E-mail: malgorzata.m.szlendak@gmail.com

Key words: colorectal neoplasms, cyclooxygenase-2, mucin 1, immunohistochemistry, pathology
Advances in systemic treatment, mainly targeted therapy and, in limited cases immunotherapy, have significantly changed the prognosis of patients with metastatic or recurrent CRC $(3,4)$. Second and subsequent lines of treatment can slow the disease and, in effect, change it into a chronic disease. However, the aim of modern oncology is to definitively eliminate the danger of disease recurrence, which might be achievable by individualization of the treatment.

Chronic inflammation is a cancer-promoting factor that leads to genetic instability (5). Cyclooxygenase-2 (COX-2) is an enzyme that catalyses the formation of prostaglandins and therefore participates in the regulation of the consecutive stages of inflammatory processes. In CRCs overexpression of COX-2 has been shown to correlate with poor survival and metastasis (6).

Mucins are a family of glycoproteins form a protective gel layer on the surface of the mucosa under normal circumstances. Their expression varies in different areas of the digestive tract and is altered in tumours. Mucins in cancer cells are dysregulated on a genetic level and atypically glycosylated in posttranscriptional setting $(7,8)$. Their quantitative and qualitative alterations impair their functions, heavily affect the properties of the mucus layer and presumably weaken the penetration of chemotherapeutic agents. Mucin 1 (MUC1) is a structural membrane-bound mucin that is normally present only on the apical borders of the secretory epithelium. In tumours, the polarization of MUC1 is lost and the protein is overexpressed at high levels over the entire cell surface (9). The positive correlation between abundant expression of MUC1 and tumour invasiveness, metastasis and poor prognosis has been reported in CRC $(9,10)$.

The overexpression of COX-2 and the overexpression of MUC1 might be related and this relationship has already been studied in pancreatic cancer (11). The authors concluded that the intracellular tail of MUC1 participates in the activation of proapoptotic genes and indirectly upregulates COX-2 expression (11). Hypothetically, this relationship may also apply to other gastrointestinal cancers, including CRC. Perhaps this could be easily observed as altered COX-2 and MUC1 expression. In addition, $\mathrm{MUC1}$ has been proposed as an alternative target for blocking COX-2 overexpression (11). In CRC, where abundant expression of COX-2 occurs frequently, such a discovery might be a substantial finding from the perspective of future targeted therapy. The significance of the present study 
was to confirm the presented hypotheses in CRC. The direct aim was to investigate the correlation between COX-2 and MUC1 expression patterns and clinical-pathological factors in $\mathrm{CRC}$, with particular attention to survival.

\section{Materials and methods}

Tissue samples. CRC samples were collected from the resources of the Histopathology Department of the Independent Clinical Hospital No. 1 (SPSK 1) in Lublin (Poland) in the form of paraffin-embedded tissue blocks. The studied material encompassed sections of colorectal adenocarcinomas from 170 patients treated in the Department of Surgical Oncology (Medical University of Lublin) between August 2004 and January 2014. Only samples that contained adenocarcinomas were included. Rectal tumour samples from patients who had undergone neoadjuvant radiotherapy were excluded from further analysis.

The clinicopathological data of patients were collected from the Medical Records Department and Outpatient Clinics of the Hospital. In the analysed population $102(60 \%)$ patients were male, the median age was 62 years, $126(74 \%)$ of the tumours were rectal cancers and the remaining 44 cases (26\%) were colon cancers. Among the disease stages there were: $8(5 \%)$ cancers in situ and 31 (18\%) stage I, 51 (30\%) stage II, $58(34 \%)$ stage III and 22 (13\%) stage IV cancers. Detailed data concerning the studied population are presented in Table I.

Antibodies. Immunostaining was performed using two antibodies for both COX-2 and MUC1. These were: Anti-COX-2 rabbit monoclonal antibody from Abcam (clone SP21, cat. no. ab16701) and anti-COX 2 mouse monoclonal antibody from Dako (clone CX-294); anti-Mucin 1C-term rabbit monoclonal antibody from antibodiesonline.com (clone G22-L, cat. no. ABIN371859) and anti-MUC1 mouse monoclonal antibody from Dako (EMA-human epithelial membrane antigen, clone E29, cat. no. M0613). The antibodies were stepwise optimized and tested at dilutions from 1:100 to 1:800 and for one antibody (anti-mucin $1 \mathrm{C}$-term clone G22-L, cat. no. ABIN371859 from antibodies online.com) even at 1:3200. The staining was comparatively performed with DAB and Bright-DAB and then the sections were counterstained with haematoxylin.

In effect, two antibodies and two staining protocols were chosen as the methods that provide the best sensitivity at the lowest background noise: Anti-COX-2 from Abcam (clone SP21, cat. no. ab16701), at a dilution of 1:200, incubated for $1 \mathrm{~h}$ and stained with DAB and anti-Mucin $1 \mathrm{C}$-term from antibodies-online.com (clone G22-L, cat. no. ABIN371859) diluted to $1: 1,000$, incubated overnight and stained with Bright-DAB (Table II).

Immunohistochemistry. Preparation of microscope slides, immunostaining and scoring were performed at University Medical Center Utrecht in the Netherlands. Sections of tissues $(4 \mu \mathrm{m})$ were first deparaffinized and blocked for endogenous peroxidase activity by immersion in $0.3 \% \mathrm{H}_{2} \mathrm{O}_{2}$ in methanol for $20 \mathrm{~min}$. Then, antigen retrieval was performed in sodium citrate buffer $(0.01 \mathrm{M} / \mathrm{pH} 6.0)$ for $20 \mathrm{~min}$ at $100^{\circ} \mathrm{C}$. Nonspecific binding sites were blocked using Protein Block
Table I. Characteristics of the study population.

\begin{tabular}{lc}
\hline Characteristic & Value \\
\hline Age, years & \\
$\leq 55, \mathrm{n}(\%)$ & $50(29.41)$ \\
$56-69, \mathrm{n}(\%)$ & $74(43.53)$ \\
$\geq 70, \mathrm{n}(\%)$ & $46(27.06$ \\
$\mathrm{M} \pm$ SD & $64.48 \pm 10.74$ \\
Me (Q1-Q3) & $62(54-70)$ \\
Min-Max & $32-87$ \\
Sex, $\mathrm{n}(\%)$ & \\
Male & $102(60.00)$ \\
Female & $68(40.00)$ \\
Localization, $\mathrm{n}(\%)$ & \\
Colon & $44(25.88)$ \\
Rectum & $126(74.12)$ \\
Stage, $\mathrm{n}(\%)$ & \\
0 & $8(4.71)$ \\
1 & $31(18.23)$ \\
2 & $51(30.00)$ \\
3 & $58(34.12)$ \\
4 & $22(12.94)$
\end{tabular}

M, mean; Me, median; Q1, lower quartile; Q3, upper quartile; Min, minimum score; Max, maximum score.

Serum Free (DAKO, X0909) for $10 \mathrm{~min}$, followed by primary antibody incubation (anti-COX-2 at room temperature for $1 \mathrm{~h}$ and anti-MUC1 at $4^{\circ} \mathrm{C}$ overnight). Antibody binding was visualized using the BrightVision+poly-HRP detection system (VWR International, cat. no. VWRKDPVB110HRP), with 3,3-diamino-benzidine as chromogen (DAB, Sigma D5637 for COX-2 and Bright-DAB, VWR International VWRKBS04-110 for MUC1). Finally, the sections were counterstained with haematoxylin, dehydrated and coverslipped using Pertex (Table II).

Analysis of immunostaining intensity was conducted at 20x original objective magnification in the area that encompassed tumour cells with the strongest staining. Scoring systems for COX-2 and MUC1 based on the references $(12,13)$. Scoring was performed independently by two researchers, the discrepancies were reanalysed by the expert and scored in accordance with his judgement.

The study was reviewed and approved by the Bioethics Committee of Medical University of Lublin (number of approval KE-0254/48/2015 from 26.02.2015).

Statistical analysis. The Statistica 9.1 software package was used for statistical analysis. The analysed data concerned the level of COX-2 and MUC1 expression with respect to age and sex of patients as well as the localization and stage of the tumour. The information regarding particular tests is included in the description of the tables.

The analysis regarding the interplay between the COX-2 and MUC1 expression levels was performed as a Spearman's rank correlation. The results were verified by the Chi-square test 
Table II. Immunohistochemistry methods.

\begin{tabular}{|c|c|c|c|c|c|}
\hline Antibody & Source & Dilution & Antigen retrieval & $\begin{array}{l}\text { Antibody } \\
\text { incubation }\end{array}$ & Chromogen \\
\hline $\begin{array}{l}\text { COX-2, clone SP21, } \\
\text { cat. no. ab16701 }\end{array}$ & Abcam & 1:200 & $\begin{array}{l}\text { Sodium citrate buffer }(0,01 \mathrm{M} / \mathrm{pH} 6.0) \\
20 \text { min at } 100^{\circ} \mathrm{C}\end{array}$ & $1 \mathrm{~h}$ & $\mathrm{DAB}$ \\
\hline $\begin{array}{l}\text { MUC1 C-term, clone G22-L, } \\
\text { cat. no. ABIN371859 }\end{array}$ & $\begin{array}{l}\text { Antibodies } \\
\text { Online }\end{array}$ & $1: 1,000$ & $\begin{array}{l}\text { Sodium citrate buffer }(0,01 \mathrm{M} / \mathrm{pH} 6.0) \\
20 \text { min at } 100^{\circ} \mathrm{C}\end{array}$ & Overnight & Bright-DAB \\
\hline
\end{tabular}

COX-2, cyclooxygenase-2; MUC1, mucin 1.

A

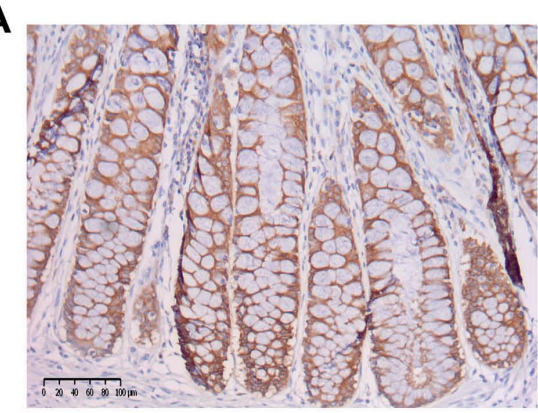

C

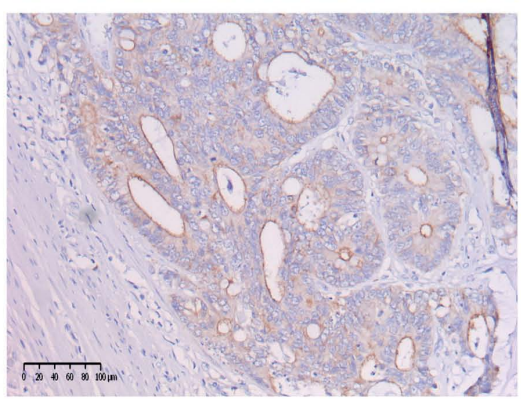

E

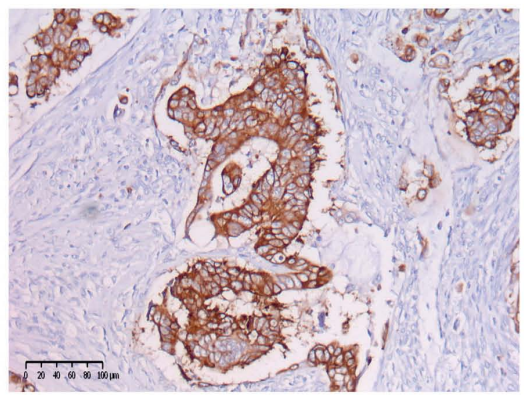

B

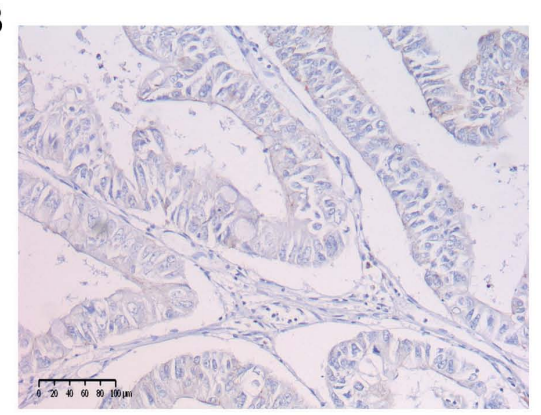

D

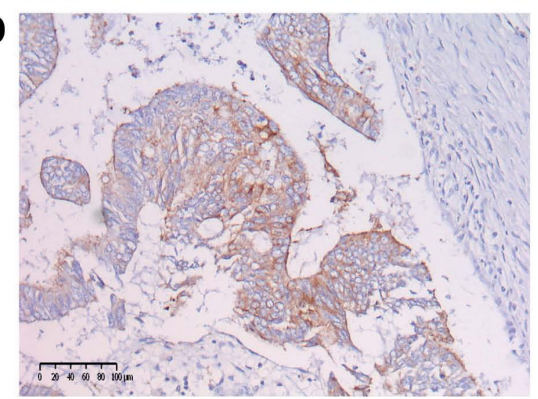

$\mathbf{F}$

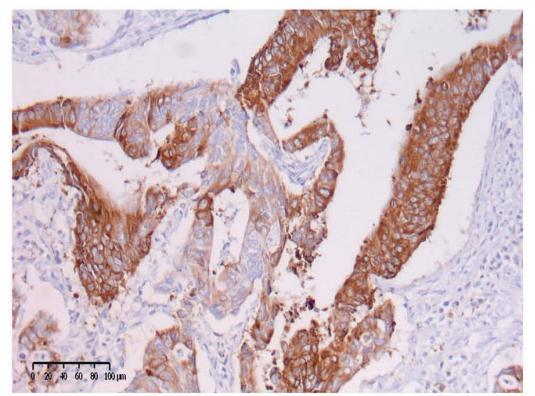

Figure 1. Immunohistochemical COX-2 staining at x200 magnification. (A) Normal colon epithelium and representative colorectal cancer samples. (B) Category 0 , no staining. (C) Category 1, weak diffuse cytoplasmic staining. (D) Category 2, moderate to strong granular cytoplasmic staining in 10-50\% of cancer cells. (E) Category 3, $>50 \%$ of tumor cells stained with a strong intensity. (F) patchy, heterogenic staining. Categories 0 and 1 were considered to demonstrate a low expression of COX-2 and scores 2 and 3, as well as patchy staining, was considered to demonstrate a high expression of COX-2. COX-2, cyclooxygenase-2.

comparing the incidence of cases with high COX-2 expression and high MUC1 expression, compared to the cases with low COX-2 expression and low MUC1 expression. An analogous analysis was performed comparing the cases with high COX-2 expression and low MUC1 expression compared to cases with low COX-2 expression and high MUC1 expression.Survival analysis was performed for high and low COX-2 expression and for high and low MUC1 expression, using MedCalc software. The results are presented as Kaplan-Meier curves, and the comparison of survival curves was performed using the log-rank test. P-values $<0.05$ were considered significant.

\section{Results}

COX-2 and MUC1 expression. COX-2 expression was categorized as follows: 0 , no staining; 1 , weak diffuse cytoplasmic staining; 2, moderate to strong granular cytoplasmic staining in $10-50 \%$ of cancer cells; and 3,>50\% of tumour cells stained with strong intensity (Fig. 1). Samples with scores of 0 and 1 were further categorized as low COX-2 expression, and those with scores of 2 and 3 were categorized as high COX-2 expression. The cases of patchy, heterogenic COX-2 staining were distinguished and included as stained with strong intensity. 
Table III. COX-2 expression and clinicopathological features of patients.

\begin{tabular}{|c|c|c|c|}
\hline \multirow[b]{2}{*}{ Characteristic } & \multicolumn{2}{|c|}{ COX-2 expression } & \multirow[b]{2}{*}{ Statistics } \\
\hline & Low, n (\%) & High, n (\%) & \\
\hline Age, years & & & $\mathrm{H}(2.170)=1.109 ; \mathrm{P}=0.574$ \\
\hline$\leq 55$ & $10(20.0)$ & $40(80.0)$ & $\mathrm{R}=0.415 ; \mathrm{P}=0.678$ \\
\hline $56-69$ & $14(18.9)$ & $60(81.1)$ & \\
\hline$\geq 70$ & $11(23.9)$ & $35(76.1)$ & \\
\hline Sex & & & $\mathrm{Z}=0.068 ; \mathrm{P}=0.945$ \\
\hline Male & $22(21.6)$ & $80(78.4)$ & \\
\hline Female & $13(19.1)$ & $55(80.9)$ & \\
\hline Localization & & & $\mathrm{Z}=0.006 ; \mathrm{P}=0.995$ \\
\hline Colon & $7(15.9)$ & $37(84.1)$ & \\
\hline Rectum & $28(22.2)$ & $98(77.8)$ & \\
\hline Stage & & & $\mathrm{H}(4.170)=1.203 ; \mathrm{P}=0.878$ \\
\hline 0 & $2(25.0)$ & $6(75.0)$ & $\mathrm{R}=0.084 ; \mathrm{P}=0.276$ \\
\hline 1 & $8(25.8)$ & $23(74.2)$ & \\
\hline 2 & $10(19.6)$ & $41(80.4)$ & \\
\hline 3 & $12(20.7)$ & $46(79.3)$ & \\
\hline 4 & $3(13.6)$ & $19(86.3)$ & \\
\hline Total & $35(20.6)$ & $135(79.4)$ & - \\
\hline
\end{tabular}

P-value, statistical significance; Z, Mann-Whitney U test score; H, Kruskal-Wallis test score; R, Spearman's rank correlation coefficient; COX-2, cyclooxygenase-2.

A
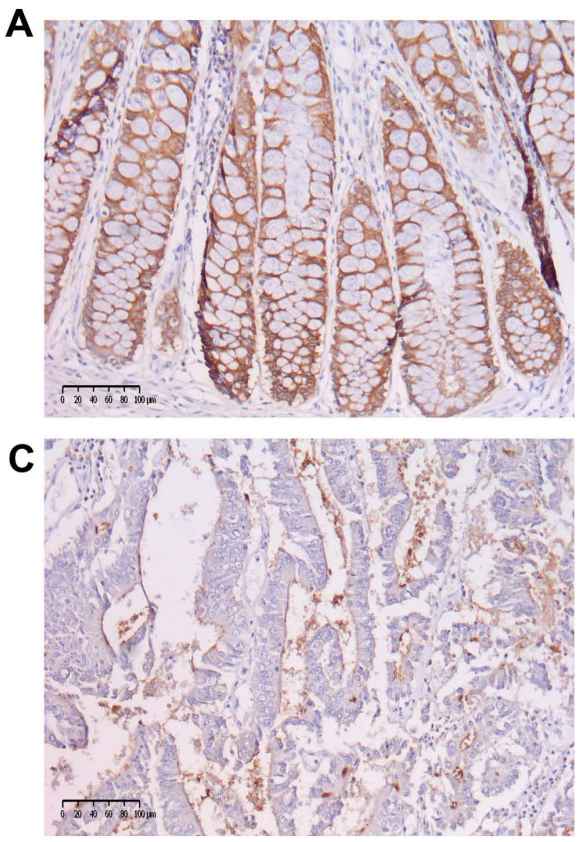

B

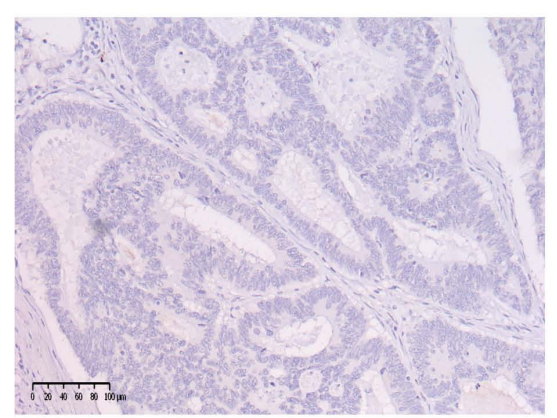

D

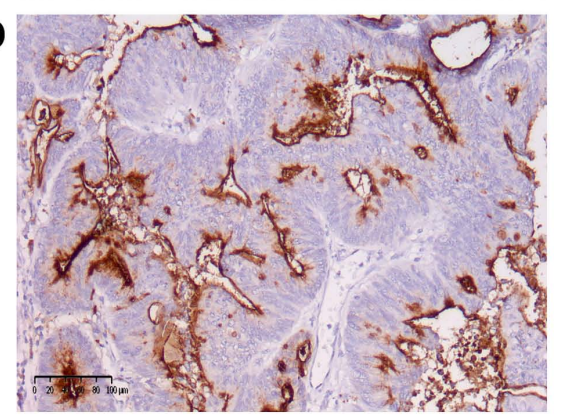

Figure 2. Immunohistochemical mucin 1 staining at x200 magnification. (A) Normal colon epithelium and representative colorectal cancer samples. (B) Category 0, no staining. (C) Category 1, weak staining. (D) Category 2, moderate to strong staining.

The expression level of COX-2 and its statistical correlation with: Age, sex, localization and disease stage are presented in Table III.

MUC1 staining was categorized as: 0, no staining; 1, weak staining; 2, strong staining and further categorized as low
MUC1 expression (scores of 0 and 1) or high MUC1 expression (score 2) (Fig. 2). The correlations with clinical data are presented in Tables III and IV.

Neither the COX-2 expression level nor the MUC1 expression level correlated with any of the clinicopathological features. 
Table IV. MUC1 expression and clinicopathological features of patients.

\begin{tabular}{|c|c|c|c|}
\hline \multirow[b]{2}{*}{ Characteristic } & \multicolumn{2}{|c|}{ MUC1 expression } & \multirow[b]{2}{*}{ Statistics } \\
\hline & Low $(0-1), \mathrm{n}(\%)$ & High (2), n (\%) & \\
\hline Age, years & & & $\mathrm{H}(2.170)=1.965 ; \mathrm{P}=0.375$ \\
\hline$\leq 55$ & $26(52.0)$ & $24(48.0)$ & $\mathrm{R}=0.107 ; \mathrm{P}=0.164$ \\
\hline $56-69$ & $32(43.2)$ & $42(56.8)$ & \\
\hline$\geq 70$ & $18(39.1)$ & $28(60.9)$ & \\
\hline Sex & & & $\mathrm{Z}=0.658 ; \mathrm{P}=0.510$ \\
\hline Male & $44(43.1)$ & $58(56.9)$ & \\
\hline Female & $32(47.1)$ & $36(52.9)$ & \\
\hline Localization & & & $\mathrm{Z}=-0.554 ; \mathrm{P}=0.580$ \\
\hline Colon & $22(50.0)$ & $22(50.0)$ & \\
\hline Rectum & $54(42.9)$ & $72(57.1)$ & \\
\hline Stage & & & $\mathrm{H}(4.170)=2.630 ; \mathrm{P}=0.622$ \\
\hline 0 & $3(37.5)$ & $5(62.5)$ & $\mathrm{R}=-0.048 ; \mathrm{P}=0.535$ \\
\hline 1 & $16(51.6)$ & $15(48.4)$ & \\
\hline 2 & $19(37.2)$ & $32(62.7)$ & \\
\hline 3 & $28(48.3)$ & $30(51.7)$ & \\
\hline 4 & $10(45.4)$ & $12(54.6)$ & \\
\hline Total & $76(44.7)$ & $94(55.3)$ & - \\
\hline
\end{tabular}

P-value, statistical significance; Z, Mann-Whitney U test score; H, Kruskal-Wallis test score; R, Spearman's rank correlation coefficient; MUC1, mucin 1.

Table V. Ratio of COX-2 to MUC1 expression.

\begin{tabular}{lllr}
\hline & \multicolumn{2}{c}{ MUC1 } \\
\cline { 2 - 3 } COX-2 & Low, n (\%) & High, n (\%) & Total \\
\hline Low & $17(10.0)^{\mathrm{a}}$ & $18(10.6)^{\mathrm{b}}$ & $35(20.6)$ \\
High & $59(34.7)^{\mathrm{c}}$ & $76(44.7)^{\mathrm{d}}$ & $135(79.4)$ \\
Total & $76(44.7)$ & $94(55.3)$ & $170(100.0)$ \\
\hline
\end{tabular}

${ }^{\mathrm{a}, \mathrm{d}}$ Chi-squared test $=36.172, \mathrm{P}<0.001 ;{ }^{\mathrm{b}, \mathrm{c}}$ Chi-squared test $=20.779$, $\mathrm{P}<0.001$. Spearman's rank correlation $\mathrm{R}-0.121, \mathrm{P}=0.117$. COX-2, cyclooxygenase-2; MUC1, mucin 1.

The ratio of COX-2 to MUC1 expression is shown in Fig. 3. There was no linear correlation between the expression levels of these proteins $(\mathrm{P}=0.117)$. However, there were significantly more cases with the simultaneous occurrence of high COX-2 and MUC1 expression compared to those in which the level of COX-2 and MUC1 expression was low $(\mathrm{P}<0.001)$. There were also significantly more cases with high expression of COX-2 and low expression of MUC1 than cases with simultaneous low expression of COX-2 and high expression of MUC1 $(\mathrm{P}<0.001)$ (Table V).

Survival analysis. There was no statistical correlation between survival and the levels of COX-2 or MUC1 expression (Figs. 4 and 5).

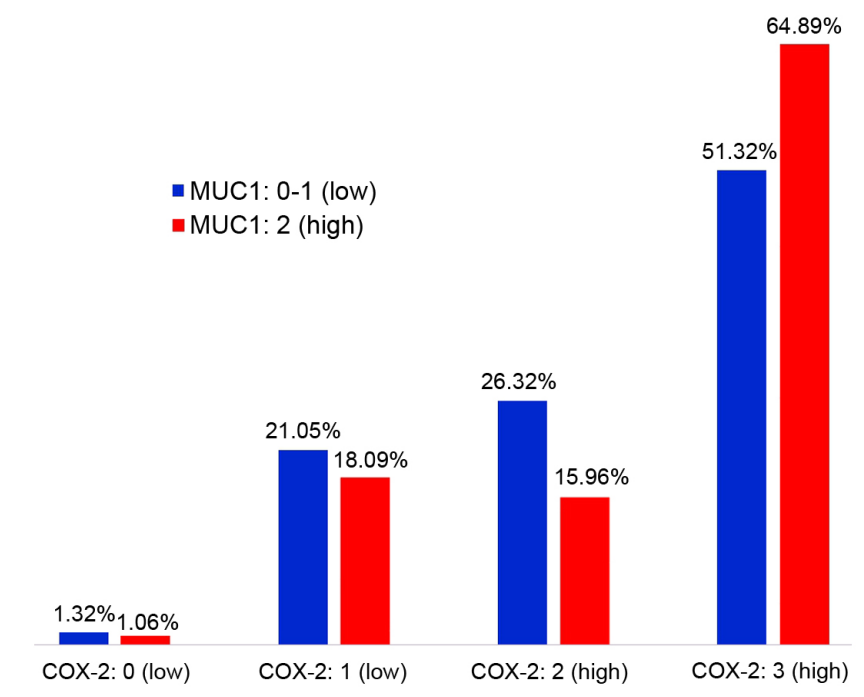

Figure 3. Ratio of COX-2 expression to MUC1 expression. COX-2, cyclooxygenase-2; MUC1, mucin 1.

\section{Discussion}

There are many reports of the importance of COX-2 overexpression in patients with CRC. Studies carried out so fa, on populations of different races, have shown that COX-2 overexpression slightly worsens overall survival (6). In a recently published paper, Kim et al presented that in a group of Korean patients elevated COX-2 expression was not a prognostic factor, but COX-2 expression might have been an 


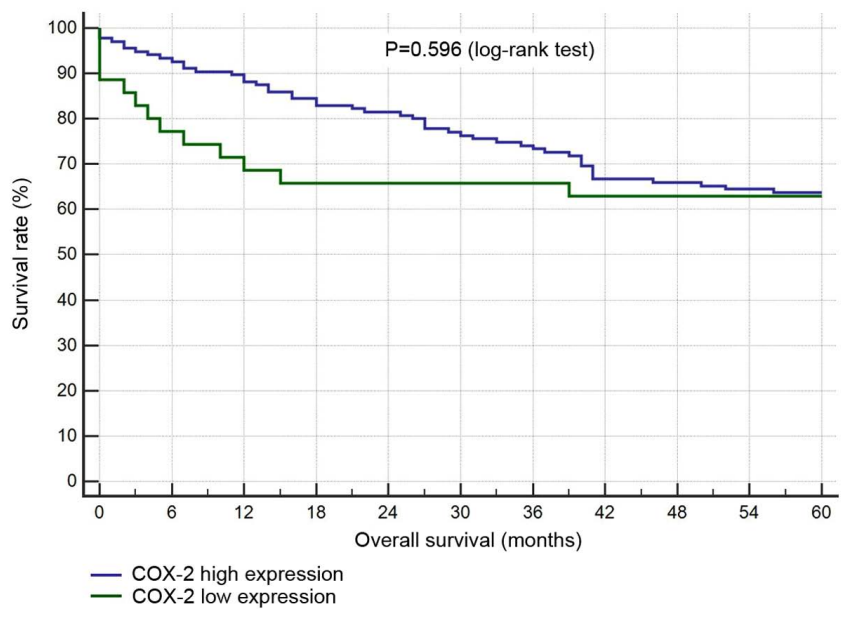

Figure 4. Survival analysis in reference to the level of COX-2 expression. COX-2, cyclooxygenase-2

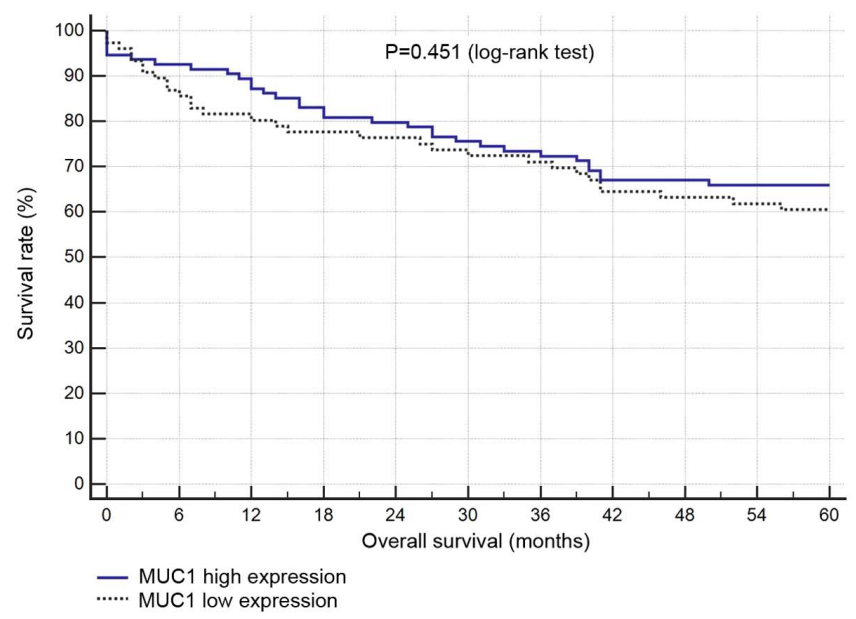

Figure 5. Survival analysis in reference to the level of MUC1 expression. MUC1, mucin 1.

independent predictive marker of late recurrence for patients with stage I to III CRC (14). Our studies showed no correlation between the expression of COX-2 and any studied clinical variables, or prognosis. The conclusions of Kim et al (14). seem to be consistent with ours, however, our analysis was narrower, because it did not relate to recurrence rates and it was also carried out among Caucasian patients so the results cannot be directly compared.

Our research did not confirm the correlation between the expression of MUC1 and clinical variables, including stage of the disease and survival. In a similar study performed independently at the same time as ours, there was no correlation between MUC1 expression and clinicopathological variables of the patients, but there was a significant increase in $M U C 1$ mRNA expression in CRC compared to healthy tissues (15). This could mean that in tumours, the level of MUC1 changes with the progression of the disease. In the cited study MUC1 expression was more often detected in patients with CRC with synchronic lymph node metastases, than in those without them (15). Duncan $e t a l$, in a study on a population of 462 patients showed that MUC1 expression can be considered an independent marker of poor prognosis, which is in contrast to our results. However, they did not confirm the correlation of MUC1 with any of the clinicopathological variables including tumour grade and stage, vascular invasion and tumour type, which coincides with our results (16). Betge et al also showed a correlation of MUC1 expression with various clinicopathological variables as well as disease progression and lymph node metastasis. However, their study did not confirm a correlation between MUC1 expression and survival in patients with CRC (17). It is interesting that all these cited studies concerned similar populations, i.e., Caucasian patients were recruited consecutively for CRC surgery. MUC1 overexpression occurs in CRC with lymph node invasion (18). Therefore, hypothetically, the negative results of our and other authors' work may result from a small number of patients with lymph node metastases.

Nevertheless, the quality assessment of the MUC1 expression level based only on immunohistochemistry is limited in credibility. This is because mucins are alternatively glycosylated in tumours (7). Evaluation of the MUC1 expression level may be understated due to the specificity of the chosen antibody. In one of the larger earlier studies, MUC1 was detected only in $32.5 \%$ of CRC specimens (19). Therefore, we take into account that in our study, COX-2 level assessment is more reliable than MUC1. The expression of mucins and associated O-glycans differ in colorectal polyp subtypes (20). Most likely, it also applies to pathological subtypes of CRC. The distribution of goblet cells, which produce mucins, increases along the entire length of the digestive tract (21). These observations might explain the discrepancies in our and other cited studies, because all of them differed in the number of pathological subtypes of CRC and the number of tumours from different localizations of the colon. In most of the papers about MUC1 in CRC, parts of the colon were not distinguished. In our study, there was a similar number of MUC1-low and MUC1-high expression in the colon, but there were definitely more cases of MUC1-high expression in the rectum. The latter result is more reliable because most of the examined tissues were cancers of the rectum.

The present study does not confirm a direct relationship between the intensity of expression of COX-2 and MUC1 or between the expression of either of them and the clinicopathological characteristics of patients with CRC. In addition, neither protein had prognostic value for survival, which contradicts some previous reports. This issue needs further investigation based on larger sample analysis or stratified analysis.

\section{Acknowledgements}

Not applicable.

\section{Funding}

The present study was supported by internal grants from the Medical University of Lublin (grant no. DS201/2018) and an educational grant for young researchers (grant no. M. Szlendak, MNsd228/2018).

\section{Availability of data and materials}

The datasets used and/or analyzed during the current study are available from the corresponding author on reasonable request. 


\section{Authors' contributions}

MS, RS and RM concieved and designed the current study. RM, RS, FM, MS, MB and JM acquired the data. MS, JM, GJAO and WPP analyzed and interpreted the data. MS and RS drafted the manuscript. All authors critically revised the manuscript, with major contributions from GJAO and WPP. All authors read and approved the final manuscript.

\section{Ethics approval and consent to participate}

The board of the Bioethics Committee of the Medical University of Lublin approved the present study protocol (approval no. KE-0254/48/2015 from February 26, 2015). Informed consent was obtained at the time of original tissue collection.

\section{Patient consent for publication}

Not applicable.

\section{Competing interests}

The authors declare that they have no competing interests

\section{References}

1. Stewart BW and Wild CP: World Cancer Report 2014. Lyon, France, IARC Press, 2014.

2. Rawla P, Sunkara T and Barsouk A: Epidemiology of colorectal cancer: Incidence, mortality, survival, and risk factors. Prz Gastroenterol 14: 89-103, 2019.

3. Ohhara Y, Fukuda N, Takeuchi S, Honma R, Shimizu Y, Kinoshita I and Dosaka-Akita H: Role of targeted therapy in metastatic colorectal cancer. World J Gastrointest Oncol 8: 642-655, 2016.

4. Kalyan A, Kircher S, Shah H, Mulcahy M and Benson A: Updates on immunotherapy for colorectal cancer. J Gastrointest Oncol 9: 160-169, 2018

5. Colotta F, Allavena P, Sica A, Garlanda C and Mantovani A: Cancer-related inflammation, the seventh hallmark of cancer : Links to genetic instability. Carcinogenesis 30: 1073-1081, 2009.

6. Peng L, Zhou Y, Wang Y, Mou H and Zhao Q: Prognostic significance of COX-2 immunohistochemical expression in colorectal cancer: A meta-analysis of the literature. PLoS One 8: e58891, 2013.

7. Skierucha M, Milne AN, Offerhaus GJ, Polkowski WP, Maciejewski R and Sitarz R: Molecular alterations in gastric cancer with special reference to the early-onset subtype. World J Gastroenterol 22: 2460-2474, 2016.

8. Terada T: An immunohistochemical study of primary signet-ring cell carcinoma of the stomach and colorectum: II. Expression of MUC1, MUC2, MUC5AC, and MUC6 in normal mucosa and in 42 cases. Int J Clin Exp Pathol 6: 613-621, 2013.
9. Niv Y: MUC1 and colorectal cancer pathophysiology considerations. World J Gastroenterol 14: 2139-2141, 2008.

10. Zeng Y, Zhang Q, Zhang Y, Lu M, Liu Y, Zheng T, Feng S, Hao M and Shi $\mathrm{H}$ : $\mathrm{MCl}$ predicts colorectal cancer metastasis: A systematic review and meta-analysis of case controlled studies. PLoS One 10: e0138049, 2015.

11. Nath S, Roy LD, Grover P, Rao S and Mukherjee P: Mucin 1 regulates Cox-2 gene in pancreatic cancer. Pancreas 44: 909-917, 2015.

12. Sitarz R, Leguit RJ, de Leng WW, Polak M, Morsink FM, Bakker O, Maciejewski R, Offerhaus GJ and Milne AN: The COX-2 promoter polymorphism-765 $\mathrm{G}>\mathrm{C}$ is associated with early-onset, conventional and stump gastric cancers. Mod Pathol 21: 685-690, 2008.

13. Eminaga O, Wei W, Hawley SJ, Auman H, Newcomb LF, Simko J, Hurtado-Coll A, Troyer DA, Carroll PR, Gleave ME, et al: MUC1 Expression by immunohistochemistry is associated with adverse pathologic features in prostate cancer: A multi-institutional study. PLoS One 11: e0165236, 2016.

14. Kim SH, Ahn BK, Paik SS and Lee KH: Cyclooxygenase-2 expression is a predictive marker for late recurrence in colorectal cancer. Gastroenterol Res Pract 2018: 7968149, 2018.

15. Kasprzak A, Siodla E, Andrzejewska M, Szmeja J, Seraszek-Jaros A, Cofta S and Szaflarski W: Differential expression of mucin 1 and mucin 2 in colorectal cancer. World J Gastroenterol 24: 4164-4177, 2018.

16. Duncan TJ, Watson NF, Al-Attar AH, Scholefield JH and Durrant LG: The role of MUC1 and MUC3 in the biology and prognosis of colorectal cancer. World J Surg Oncol 5: 31, 2007.

17. Betge J, Schneider NI, Harbaum L, Pollheimer MJ, Lindtner RA, Kornprat P, Ebert MP and Langner C: MUC1, MUC2, MUC5AC, and MUC6 in colorectal cancer: Expression profiles and clinical significance. Virchows Arch 469: 255-265, 2016

18. Xu F, Liu F, Zhao H, An G and Feng G: Prognostic significance of mucin antigen MUC1 in various human epithelial cancers: A meta-analysis. Medicine (Baltimore) 94: e2286, 2015.

19. Baldus SE, Monig SP, Hanisch FG, Zirbes TK, Flucke U, Oelert S, Zilkens G, Madejczik B, Thiele J, Schneider PM, et al: Comparative evaluation of the prognostic value of MUC1, MUC2, sialyl-Lewis(a) and sialyl-Lewis(x) antigens in colorectal adenocarcinoma. Histopathology 40: 440-449, 2002.

20. Krishn SR, Kaur S, Sheinin YM, Smith LM, Gautam SK, Patel A, Jain M, Juvvigunta V, Pai P, Lazenby AJ, et al: Mucins and associated $\mathrm{O}$-glycans based immunoprofile for stratification of colorectal polyps: Clinical implication for improved colon surveillance. Oncotarget 8: 7025-7038, 2016.

21. Kim JJ and Khan WL: Goblet cells and mucins: Role in innate defense in enteric infections. Pathogens 2: 55-70, 2013.

This work is licensed under a Creative Commons Attribution-NonCommercial-NoDerivatives 4.0 International (CC BY-NC-ND 4.0) License. 